\title{
A Robust Optimization Approach to Energy and Reserve Dispatch in Electricity Markets
}

\section{Zugno, Marco; Conejo, Antonio J.}

Publication date:

2013

Document Version

Publisher's PDF, also known as Version of record

Link back to DTU Orbit

Citation (APA):

Zugno, M., \& Conejo, A. J. (2013). A Robust Optimization Approach to Energy and Reserve Dispatch in Electricity Markets. Technical University of Denmark. Technical Report-2013 No. 05

\section{General rights}

Copyright and moral rights for the publications made accessible in the public portal are retained by the authors and/or other copyright owners and it is a condition of accessing publications that users recognise and abide by the legal requirements associated with these rights.

- Users may download and print one copy of any publication from the public portal for the purpose of private study or research.

- You may not further distribute the material or use it for any profit-making activity or commercial gain

- You may freely distribute the URL identifying the publication in the public portal

If you believe that this document breaches copyright please contact us providing details, and we will remove access to the work immediately and investigate your claim 


\title{
A Robust Optimization Approach to Energy and Reserve Dispatch in Electricity Markets
}

\author{
Marco Zugno $^{a, *}$, Antonio J. Conejo ${ }^{b}$ \\ ${ }^{a}$ Department of Applied Mathematics and Computer Science, Technical University of Denmark \\ ${ }^{b}$ Department of Electrical Engineering, University of Castilla-La Mancha
}

\begin{abstract}
To a large extent, electricity markets worldwide still rely on deterministic procedures for clearing energy and reserve auctions. However, larger and larger shares of the production mix consist of renewable sources whose nature is stochastic and non-dispatchable, as their output is not known with certainty and cannot be controlled by the operators of the production units. Stochastic programming models for the joint determination of the day-ahead energy and reserve dispatch, necessary for coping with the real-time output deviations from these sources, have been proposed in the literature. In this work, we take an alternative approach and cast the problem as an adaptive robust optimization problem. The day-ahead and reserve schedules determined in this fashion yield the minimum system cost, accounting for the cost of the redispatching decisions at the balancing stage, in the worst-case realization of the stochastic production within a specified uncertainty set. In a case-study based on a 24-node system, we assess the degree of suboptimality of the robust solution with respect to the optimal dispatch obtained with a stochastic programming approach, and compare their worst-case cost. Furthermore, we discuss the robustness of these two alternative approaches with respect to changes in the distribution of the uncertainty, as well as their computational properties.
\end{abstract}

Keywords: OR in energy, Robust Optimization, Decomposition, Electricity market, Reserve market, Renewable Energy

\section{Nomenclature}

\section{Decision Variables}

$\mathbf{x}$ Vector of decision variables at the dispatching stage, including energy dispatch, upward and downward reserve, and network state variables at the nodes of the system;

$\Delta \mathbf{w}$ Forecast error for stochastic power production;

\footnotetext{
* Contact author:

Technical University of Denmark

Department of Applied Mathematics and Computer Science

Matematiktorvet, Building 322, DK-2800 Kgs. Lyngby, Denmark

Tel: +45 4525 3369, email: mazu@dtu.dk
} 
y Vector of decision variables at the balancing stage, including energy redispatch, load shedding, stochastic power spillage and actual state variables at the nodes of the system.

\section{Parameters}

$\mathbf{c}_{\mathbf{x}}$ Coefficients in the cost function associated with the day-ahead dispatching (energy and reserve);

$\mathbf{c}_{\mathbf{y}}$ Coefficients in the cost function associated with the redispatching at the balancing stage;

d Demand at each node of the system (considered known with certainty);

$\widehat{\mathrm{w}}$ Conditional expectation of stochastic power production.

\section{Introduction}

In recent years, renewable electricity production sources have experienced an unprecedented growth in installed capacity worldwide. Such a development is explained both by technological advance, which has made production from sources like wind and solar cheaper, and by governmental support aimed at promoting sustainability. Sources of this type are fundamentally different from conventional means of electricity generation. Indeed, they are stochastic, i.e., their production is not known with certainty in advance, and non-dispatchable, i.e., the power plant operators have partial or no control on the output level.

Owing to the features described above, an increasing penetration of renewables challenges the traditional way electricity markets are operated. In particular, deterministic schemes have been employed for years to assess the amount of reserve capacity, the availability of which is needed in order to cope with unforeseen events in the system. Typically, mechanisms for reserve determination are based on deterministic $N-1$ or $N-k$ criteria, which guarantee the functioning of the system in the event of loss of the largest unit, or of the $k$ largest units in the system, respectively. As the penetration of stochastic generation sources in power system grows, reserves are increasingly used to cover the fluctuations of the power output from renewables, thus calling for stochastic decision-making tools.

In parallel, market-clearing procedures for energy markets are also challenged by the growth of stochastic production capacity. For example, the day-ahead market is cleared according to a deterministic leastcost dispatch principle based on the offers and bids submitted by suppliers and consumers. Stochastic production is normally dispatched at a point forecast of its output distribution. However, this procedure does not account for the projected cost of the dispatch at the balancing stage, and is therefore suboptimal, see [10].

The existing literature on the subject shows that system cost can be significantly reduced in expectation by jointly optimizing the day-ahead dispatch and the reserve in a stochastic programming framework, see [9]. However, models of this type require that the market operator has an accurate probabilistic description of the joint distribution of uncertain production at different locations in the grid, which is far from trivial. Furthermore, such models may require unreasonable solution time as the discrete number of scenarios used to approximate the distribution of the uncertainty increases.

An alternative framework to stochastic programming for dealing with problems under uncertainty is robust optimization. In this framework, stochastic variables are assumed to take values within an uncertainty set. Then, a robust decision is determined as the solution to an optimization problem that must be feasible for any realization of the uncertainty, and optimal in the worst-case choice of the stochastic parameters in the aforementioned set. The modeling effort is reduced in robust optimization to a description of a meaningful set over which the uncertain parameters may take values in, i.e., the support of the density function rather than the full probability distribution required by the stochastic programming approach.

The equivalent of stochastic programming with recourse in robust optimization is adaptive robust optimization. This framework aims at minimizing the total cost in the worst-case realization of the uncer- 
tainty, assuming that recourse actions can be taken as a response to the realization of the uncertainty. For an introduction to robust optimization, and to its adaptive version, we refer the interested reader to $[1]$.

Recent applications of adaptive robust optimization focusing on electricity markets are presented in [2], [7] and [14], which study the unit commitment problem under uncertain load or production from stochastic sources. In all these works, a cutting-plane approach is employed to exploit the convex dependence of the objective function on the first-stage decision, see [12]. Then, the resulting max-min problem resulting from the sequential enforcement of the worst-case (maximum-cost) parameter realization and decision on the optimal recourse action is cast as a single-level bilinear program. This problem is then solved either with an outer-approximation technique [2], or as a Mixed Integer Linear Program (MILP) after linearization through binary expansion $[7,14]$.

The main features of the model presented in this paper are threefold:

1. We apply adaptive robust optimization to the problem of determining the optimal day-ahead energy and reserve dispatch in a single time-period, rather than to unit commitment. The problem we consider is particularly interesting as it resembles the current design of electricity markets in Europe, where decisions on unit commitment are left to the power producers, and the market operator determines sequentially the amount of reserves needed and the optimal day-ahead energy dispatch. Note that the extension of the proposed model to a multi-period setting does not entail any conceptual complication. We propose the joint determination of day-ahead dispatch and reserve akin to the one in [9], though in a robust optimization framework.

2. We propose a reformulation of the inner max-min problem for the determination of the uncertainty and recourse decision that can accommodate general polyhedral sets for the uncertainty and converges to an exact solution. Our approach is more flexible than the binary reformulation in [7] and [14] in that it allows to model any polyhedral uncertainty set, still using a comparable number of binary variables. In comparison to the approach in [2], which is more flexible with respect to the choice of the uncertainty set, our reformulation guarantees convergence to an exact solution.

3. We compare the results obtained from the robust optimization approach with the ones from the corresponding stochastic programming version of the model.

The structure of the paper is the following. In Section 2, we introduce the formulation of the problem. Algorithms for solving this problem are then described in Section 3. Then, Section 4 presents results from a simple illustrative example and from a larger case study based on the 24-node IEEE Reliability Test-System in [6]. Finally, conclusions are presented in Section 5.

\section{Problem Formulation}

When an electric energy system includes stochastic production sources, the joint determination of the optimal day-ahead energy and reserve dispatch can be formulated as the following problem of optimization under uncertainty:

$$
\begin{array}{ll}
\min _{\mathbf{x}} & \mathbf{c}_{\mathbf{x}}^{T} \mathbf{x}+\mathcal{Q}_{\mathcal{W}}(\mathbf{x}) \\
\text { s.t. } & \mathbf{F} \mathbf{x}=\mathbf{d}-\widehat{\mathbf{w}} \\
& \mathbf{G x} \geq \mathbf{g}
\end{array}
$$

The vector $\mathbf{x}$ of decision variables includes energy dispatch, upward and downward reserve as well as state variables at each node of the transmission network.

For the sake of clarity, we split the set of constraints into two groups. Group (1b) only includes equalities, which represent the balancing conditions. Such constraints guarantee that for each node of the transmission network, the day-ahead energy dispatch for production and the net power inflow, which are 
either a subset or linearly dependent on a subset of the variables $\mathbf{x}$, are equal to the net demand, i.e., consumption, $\mathbf{d}$, minus the forecast stochastic power production, $\widehat{\mathbf{w}}$. The second set of constraints (1c) includes upper and lower bounds to the energy and reserve dispatch, to the scheduled power flows, as well as declarations of nonnegative variables.

The objective function (1a) is equal to the sum of the cost associated with the day-ahead decision $\mathbf{c}_{\mathbf{x}}{ }^{T} \mathbf{x}$ and a measure of the stochastic optimal recourse cost, $\mathcal{Q}_{\mathcal{W}}(\mathbf{x})$. Such a measure is a function of the first-stage decision only, and is parameterized on the distribution $\mathcal{W}$ of the uncertainty, in this case stochastic power generation. In stochastic programming, typical choices of $\mathcal{Q}_{\mathcal{W}}(\mathbf{x})$ are the expectation or the conditional value at risk (or a combination of these) over a discrete set of scenarios approximating the actual distribution of the uncertainty. In a robust optimization framework, instead, we seek to minimize the recourse cost in the worst-case realization of the stochastic parameters within an uncertainty set. The determination of the worst-case recourse cost, or redispatch cost, writes as the following max-min programming problem, parameterized on the first-stage decision $\mathbf{x}$ :

$$
\begin{array}{cc}
\mathcal{Q}_{\mathcal{W}}(\mathbf{x})=\max _{\boldsymbol{\Delta} \mathbf{w}} \min _{\mathbf{y}} \mathbf{c}_{\mathbf{y}}{ }^{T} \mathbf{y} & \\
\text { s.t. } \mathbf{P y}=-\boldsymbol{\Delta} \mathbf{w}-\mathbf{Q x}, & : \boldsymbol{\lambda}, \\
\mathbf{L} \mathbf{y} \geq \mathbf{l}-\mathbf{M} \mathbf{x}-\mathbf{N} \boldsymbol{\Delta} \mathbf{w}, & : \boldsymbol{\mu}, \\
\text { s.t. } \mathbf{H} \boldsymbol{\Delta} \mathbf{w} \leq \mathbf{h} . &
\end{array}
$$

Model (2) has a max-min structure that allows the determination of the minimum recourse cost in the worst-case realization of the uncertainty. Indeed, the maximization problem chooses the worst-case realization of the stochastic deviation $\Delta \mathrm{w}$ of stochastic power production from the conditional mean forecast within the polyhedral uncertainty set defined by the set $(2 \mathrm{~d})$ of linear inequalities. After the worst-case realization of the uncertainty is chosen, the recourse cost is minimized in the min problem in (2a). The vector $\mathbf{y}$ of optimization variables of this problem consists of the energy redispatch for each producer, load shedding, spillage of stochastic production and the network state variable at each node in the balancing stage. The set of equations (2b) ensure that redispatch plus additional net flow equals the error of stochastic power prediction at each node. Notice that (2b) depends on the first-stage network state variables included in the day-ahead decision vector $\mathbf{x}$. Furthermore, the problem is constrained by the set (2c) of linear inequalities, which include upper and lower bounds on energy redispatch, load shedding, stochastic power spillage, actual power flows and declarations of nonnegative variables. Notice that reserve, which is included in the day-ahead decision vector $\mathbf{x}$, limits the provision of backup power at the recourse stage. Furthermore, stochastic power spillage is limited above by the actual stochastic power production $\widehat{\mathbf{w}}+\Delta \mathrm{w}$.

\subsection{Reformulation as a Min-Max Bilinear Problem}

The formulation in the above section cannot be immediately employed in practice. Indeed, the direct substitution of $\mathcal{Q}_{\mathcal{W}}(\mathbf{x})$ as defined in (2) results in a min-max-min problem, for which a general-purpose solution algorithm is not available. However, following the derivations in [2], [7] and [14], it is possible to reformulate it as a min-max problem by substituting the right-hand side max problem with its dual. This results in the following formulation:

$$
\begin{gathered}
\min _{\mathbf{x}} \mathbf{c}_{\mathbf{x}}^{T} \mathbf{x}+\max _{\boldsymbol{\Delta} \mathbf{w}} \max _{\boldsymbol{\lambda}, \boldsymbol{\mu}}(-\boldsymbol{\Delta} \mathbf{w}-\mathbf{Q} \mathbf{x})^{T} \boldsymbol{\lambda}+(\mathbf{l}-\mathbf{M} \mathbf{x}-\mathbf{N} \boldsymbol{\Delta} \mathbf{w})^{T} \boldsymbol{\mu} \\
\text { s.t. } \mathbf{P}^{T} \boldsymbol{\lambda}+\mathbf{L}^{T} \boldsymbol{\mu}=\mathbf{c}_{\mathbf{y}}, \\
\boldsymbol{\mu} \geq 0, \\
\text { s.t. } \mathbf{H} \boldsymbol{\Delta} \mathbf{w} \leq \mathbf{h}, \\
\text { s.t. } \mathbf{F} \mathbf{x}=\mathbf{d}-\widehat{\mathbf{w}}, \\
\mathbf{G} \mathbf{x} \geq \mathbf{g} .
\end{gathered}
$$

It should be noticed that model (3) is in fact a min-max programming problem. Indeed, the midand right-hand-side maximization problems can be merged into a single maximization problem in the 
optimization variables $\boldsymbol{\Delta} \mathbf{w}, \boldsymbol{\lambda}$ and $\boldsymbol{\mu}$. Furthermore, we remark that the optimization problem resulting from this merging is a bilinear one, as it involves cross-products between $\Delta \mathbf{w}$ and the lower-level decision variables $\boldsymbol{\lambda}$ and $\boldsymbol{\mu}$ in the objective function (3a).

We conclude the section with the following two observations, which will turn out useful in Section 3.

1. The problem resulting from the merging of the mid and the right-hand-side maximization problems (3a)-(3c) is bilinear and defined over a polyhedral set. As a consequence, its optimal solution is one of the vertices of this set.

2. Since the vector $\mathbf{x}$ of day-ahead decision variables only appears in the objective function and not in the constraints, the feasible polyhedron is independent of the day-ahead decision, and hence it has a finite number of vertices.

\subsection{Reformulation as a Linear Min-Max Problem with Equilibrium Con- straints}

Let us consider again formulation (3). We notice that it is possible to swap the order of the mid and right-hand-side maximization problems, thus first optimizing over the variables $\boldsymbol{\lambda}, \boldsymbol{\mu}$, and then over $\boldsymbol{\Delta}$. Furthermore, not all the terms of the objective function depend on $\Delta \mathbf{w}$, and the constraints are separable for the two sets of variables. As a result, we can reformulate (3) as follows:

$$
\begin{aligned}
& \min _{\mathbf{x}} \mathbf{c}_{\mathbf{x}}^{T} \mathbf{x}+\max _{\boldsymbol{\lambda}, \boldsymbol{\mu}}-(\mathbf{Q} \mathbf{x})^{T} \boldsymbol{\lambda}+(\mathbf{l}-\mathbf{M} \mathbf{x})^{T} \boldsymbol{\mu}+\max _{\boldsymbol{\Delta} \mathbf{w}}-\left(\boldsymbol{\lambda}^{T}+\boldsymbol{\mu}^{T} \mathbf{N}\right) \boldsymbol{\Delta} \mathbf{w} \\
& \text { s.t. } \mathbf{H} \boldsymbol{\Delta} \mathbf{w} \leq \mathbf{h}, \\
& \text { s.t. } \mathbf{P}^{T} \boldsymbol{\lambda}+\mathbf{L}^{T} \boldsymbol{\mu}=\mathbf{c}_{\mathbf{y}}, \\
& \quad \boldsymbol{\mu} \geq 0 \\
& \text { s.t. } \mathbf{F} \mathbf{x}=\mathbf{d}-\widehat{\mathbf{w}} \\
& \mathbf{G} \mathbf{x} \geq \mathbf{g},
\end{aligned}
$$

where we indicate with $\boldsymbol{\xi}$ the set of dual variables relative to constraints $(4 \mathrm{~b})$ for the right-hand-side maximization problem.

The max-max programming problem comprising the mid and the right-hand-side optimization problems in (4) can be cast as a Mathematical Program with Equilibrium Constraints (MPEC), see [5]. However, before doing so, we point out that since the right-hand-side maximization problem is linear, the strong duality theorem holds. Therefore, the following relationship holds at optimality:

$$
-\left(\boldsymbol{\lambda}^{T}+\boldsymbol{\mu}^{T} \mathbf{N}\right) \boldsymbol{\Delta} \mathbf{w}=\mathbf{h}^{T} \boldsymbol{\xi}
$$

Differently from the term on the left-hand side of (5), the one on the right-hand side is linear, as it does not involve cross-products between optimization variables. Therefore, considering (4) and (5) renders the problem below whose objective function is linear in the optimization variables:

$$
\begin{aligned}
& \min _{\mathbf{x}} \mathbf{c}_{\mathbf{x}}^{T} \mathbf{x}+\max _{\boldsymbol{\lambda}, \boldsymbol{\mu}, \boldsymbol{\Delta} \mathbf{w}, \boldsymbol{\xi}}-(\mathbf{Q x})^{T} \boldsymbol{\lambda}+(\mathbf{l}-\mathbf{M} \mathbf{x})^{T} \boldsymbol{\mu}+\mathbf{h}^{T} \boldsymbol{\xi} \\
& \text { s.t. } \mathbf{0} \leq \boldsymbol{\xi} \perp \mathbf{h}-\mathbf{H} \boldsymbol{\Delta} \mathbf{w} \geq \mathbf{0}, \\
& \\
& \mathbf{H}^{T} \boldsymbol{\xi}=-\boldsymbol{\lambda}-\mathbf{N}^{T} \boldsymbol{\mu}, \\
& \\
& \mathbf{P}^{T} \boldsymbol{\lambda}+\mathbf{L}^{T} \boldsymbol{\mu}=\mathbf{c}_{\mathbf{y}}, \\
& \quad \boldsymbol{\mu} \geq \mathbf{0}, \\
& \text { s.t. } \mathbf{F x}=\mathbf{d}-\widehat{\mathbf{w}}, \\
& \mathbf{G x} \geq \mathbf{g} .
\end{aligned}
$$

Notice that formulations (1)-(2), (3) and (6) are equivalent. 


\section{Solution Algorithm}

In this section, we present two iterative schemes to solve the min-max-min problem (1)-(2), both of which are based on the cutting-plane algorithm in [8] within a Benders' decomposition scheme [3].

\subsection{Benders-Dual Cutting-Plane Algorithm}

Let us consider reformulation (3). Because of the observations in the last paragraph of Section 2.1, the solution to the inner level bilinear maximization problem belongs to a set of finite cardinality $K$, which does not depend on the first-stage decisions. Indicating the elements of this set as $\left(\boldsymbol{\Delta} \mathbf{w}_{k}, \boldsymbol{\lambda}_{k}, \boldsymbol{\mu}_{k}\right)$, with $k=1, \ldots, K$, we can alternatively reformulate problem (1)-(2) as follows:

$$
\begin{aligned}
\min _{\mathbf{x}, \beta} & \mathbf{c}_{\mathbf{x}}^{T} \mathbf{x}+\beta \\
\text { s.t. } & \beta \geq-\boldsymbol{\Delta} \mathbf{w}_{k}^{T} \boldsymbol{\lambda}_{k}+\left(\mathbf{l}-\mathbf{N} \boldsymbol{\Delta} \mathbf{w}_{k}\right)^{T} \boldsymbol{\mu}_{k}-\left(\boldsymbol{\lambda}_{k} \mathbf{Q}+\boldsymbol{\mu}_{k} \mathbf{M}\right) \mathbf{x}, \\
& \mathbf{F x}=\mathbf{d}-\widehat{\mathbf{w}}, \\
& \mathbf{G x} \geq \mathbf{g} .
\end{aligned}
$$

As one can see, the term $\beta$ in the objective function is bounded from below by the pointwise maximum of a finite set of linear functions in the first-stage decision variables $\mathbf{x}$. This implies that the optimal objective function value of (7) is a convex, piecewise linear function in $\mathbf{x}$. The following cutting-plane algorithm, proposed for robust optimization problems with recourse by [12], is guaranteed to converge to the optimal solution in a finite number of steps.

1. Set upper and lower bounds $\mathrm{UB}=+\infty$ and $\mathrm{LB}=-\infty$, and initialize the iteration index $i \leftarrow 1$.

2. Define the relaxed master problem (MP) as the minimization of (7a), subject to (7c) and (7d), and fix a reasonable lower bound for $\beta$, i.e., lower than the expected objective value of the inner problem. Fix a feasible solution $\left(\mathbf{x}_{1}^{*}, \beta_{1}^{*}\right)$ to the relaxed MP.

3. Solve either the following subproblem

$$
\begin{aligned}
\mathrm{SP}_{1}: \max _{\boldsymbol{\Delta} \mathbf{w}, \boldsymbol{\lambda}, \boldsymbol{\mu}} & \left(-\boldsymbol{\Delta} \mathbf{w}-\mathbf{Q x}_{i}^{*}\right)^{T} \boldsymbol{\lambda}+\left(\mathbf{l}-\mathbf{M} \mathbf{x}_{i}^{*}-\mathbf{N} \boldsymbol{\Delta} \mathbf{w}\right)^{T} \boldsymbol{\mu} \\
\text { s.t. } \quad & \mathbf{P}^{T} \boldsymbol{\lambda}+\mathbf{L}^{T} \boldsymbol{\mu}=\mathbf{c}_{\mathbf{y}}, \\
& \boldsymbol{\mu} \geq 0, \\
& \mathbf{H} \boldsymbol{\Delta} \mathbf{w} \leq \mathbf{h},
\end{aligned}
$$

or

$$
\begin{aligned}
\mathrm{SP}_{2}: \max _{\boldsymbol{\lambda}, \boldsymbol{\mu}, \boldsymbol{\Delta \mathbf { w }}, \boldsymbol{\xi}} & -\left(\mathbf{Q} \mathbf{x}_{i}^{*}\right)^{T} \boldsymbol{\lambda}+\left(\mathbf{l}-\mathbf{M} \mathbf{x}_{i}^{*}\right)^{T} \boldsymbol{\mu}+\mathbf{h}^{T} \boldsymbol{\xi} \\
\text { s.t. } \quad & \mathbf{0} \leq \boldsymbol{\xi} \perp \mathbf{h}-\mathbf{H} \boldsymbol{\Delta} \mathbf{w} \geq \mathbf{0} \\
& \mathbf{H}^{T} \boldsymbol{\xi}=-\boldsymbol{\lambda}-\mathbf{N}^{T} \boldsymbol{\mu} \\
& \mathbf{P}^{T} \boldsymbol{\lambda}+\mathbf{L}^{T} \boldsymbol{\mu}=\mathbf{c}_{\mathbf{y}} \\
& \boldsymbol{\mu} \geq \mathbf{0}
\end{aligned}
$$

Notice that (8) and (9) correspond to the merging of the mid and right-hand-side optimization problems in (3), and to the right-hand-side problem in (6), respectively, where the first-stage variables are fixed. Let us indicate the optimal SP objective function value as $z_{i}^{\text {SP* }}$. Update the upper bound $\mathrm{UB}=\min \left\{\mathrm{UB}, \mathbf{c}_{\mathbf{x}} \mathbf{x}_{i}^{*}+z_{i}^{\mathrm{SP}}\right\}$. Add to the relaxed MP the Benders cut $(7 \mathrm{~b})$ corresponding to the SP solution $\left(\Delta \mathbf{w}_{i}^{*}, \boldsymbol{\lambda}_{i}^{*}, \boldsymbol{\mu}_{i}^{*}\right)$ determined at this stage.

4. Solve the relaxed MP, fix $\mathbf{x}_{i+1}^{*}$ at the solution and $z_{i+1}^{\mathrm{MP}}$ at the objective function value. Update $\mathrm{LB}=z_{i+1}^{\mathrm{MP}}$. 
5. If $\mathrm{UB}-\mathrm{LB}<\tau$, where $\tau$ is a small tolerance value, then stop. Otherwise, update $i \leftarrow i+1$ and go back to 3 .

The above approach is named Benders-dual cutting plane algorithm in [13], since the cuts generated in step 3 of the algorithm above are based on the optimal dual solution of the lower-level problem in (2).

\subsection{Primal Cut Algorithm}

Similarly to the previous approach, the primal cut algorithm [13] is based on the fact that the solution to the lower-level optimization problem in (3) is at one of the vertices of the feasibility set. Because the feasibility set for $\Delta \mathrm{w}$ is completely decoupled from the feasibility set of the variables $(\boldsymbol{\lambda}, \boldsymbol{\mu})$, the worst-case realization of the uncertainty $\Delta \mathbf{w}$ is at a vertex of its feasibility set defined by $\mathbf{H} \boldsymbol{\Delta} \mathbf{w} \leq \mathbf{h}$.

We indicate with $\Delta \mathbf{w}_{k}$ the vertices of the feasibility set for the uncertain stochastic power production $\Delta \mathbf{w}$, and assign a copy $\mathbf{y}_{\mathbf{k}}$ of the vector of recourse decision variables to each of these vertices. Hence, one can reformulate problem (1)-(2) in the following way:

$$
\begin{array}{rlr}
\min _{\mathbf{x}, \mathbf{y}_{k}, \beta} & \mathbf{c}_{\mathbf{x}}{ }^{T} \mathbf{x}+\beta & \\
\text { s.t. } & \beta \geq \mathbf{c}_{\mathbf{y}}{ }^{T} \mathbf{y}_{k}, & \forall k, \\
& \mathbf{P y}_{k}=-\mathbf{\Delta} \mathbf{w}_{k}-\mathbf{Q x}, & \forall k, \\
& \mathbf{L} \mathbf{y}_{k} \geq \mathbf{l}-\mathbf{M} \mathbf{x}-\mathbf{N} \mathbf{\Delta} \mathbf{w}_{k}, & \forall k, \\
& \mathbf{F x}=\mathbf{d}-\widehat{\mathbf{w}}, & \\
& \mathbf{G x} \geq \mathbf{g} . &
\end{array}
$$

Notice that cuts (10b) are affine in the primal recourse variables $\mathbf{y}_{\mathbf{k}}$, as opposed to cuts $(7 \mathrm{~b})$, which depend on the dual variables. Besides, we remark that there is a copy $\mathbf{y}_{k}$ of the recourse variables and of constraints (10b), (10c) and (10d) for each vertex $\boldsymbol{\Delta} \mathbf{w}_{k}$.

The following comments are in order:

- Differently from (7), model (10) includes energy redispatch, load shedding, stochastic power spillage, and network state variables at the balancing stage as decision variables. Furthermore, there is one such set of decisions for each vertex of the uncertainty set.

- The number of vertices, and therefore of Benders cuts, in (10) is smaller than that in (7). This is because in (10) there is one Benders cut per vertex of the uncertainty set, and not per vertex of the joint feasibility set of $(\boldsymbol{\Delta} \mathbf{w}, \boldsymbol{\lambda}, \boldsymbol{\mu})$, which has higher dimensionality.

- On the other hand, the increase in size of the relaxed MP in a Benders scheme is much larger every time a vertex is added to the MP. Indeed, this entails adding a set of balancing market variables (recourse for a specific realization of the uncertainty), as well as a Benders cut (10b) and a set of feasibility constraints (10c)-(10d) for the balancing stage.

The primal cut algorithm is akin to the one described in Section 3.1, with the following differences: the master problem used in step 2 is (10); at the end of step 3, we add a set $\mathbf{y}_{\mathbf{k}}$ of recourse variables and a set of constraints (10b)-(10d), where we fix $\Delta \mathrm{w}$ at the current solution $\Delta \mathbf{w}_{i}^{*}$ of the subproblem.

As documented in [13] and [14], the primal cut algorithm, which is the one used in this work, generally guarantees a faster convergence to the optimal solution than the Benders-dual cutting plane one.

\subsection{Choice of the Subproblem and of its Solution Method}

In the description of the cutting-plane algorithm in Section 3.1, we left open the choice of the subproblem and of relative solution method in step 3 . 
To our knowledge, the existing literature on the topic exclusively considers subproblem (8). The solution of this optimization problem is far from being trivial, since the presence of bilinear terms in the objective function (3a) renders the problem non-convex. In [2], an outer-approximation algorithm is proposed to solve this problem with general uncertainty sets. However, since the subproblem is non-convex, only local convergence is guaranteed for this method. As an alternative, exact linearization methods based on the use of integer variables are proposed in [7] and [14]. However, the uncertainty set is restricted to the particular case of polyhedral budgeted sets.

In this work, we propose the use of subproblem (9). The only nonlinearity in this problem is the presence of complementarity conditions (9b), where the $\perp$ operator implies that $\boldsymbol{\xi}(\mathbf{h}-\mathbf{H} \boldsymbol{\Delta} \mathbf{w})=\mathbf{0}$. Notice that complementarity conditions can be linearized by making use of binary variables as proposed by [4]. Alternatively, one could employ the approach of [11], which is based on SOS-1 variables. In this work, the former implementation is chosen.

In comparison to the models in [7], [14] and [2], the proposed model has the following characteristics:

1. As compared to the method in [2], our approach converges to the global optimal solution. However, the model in [2] considers general convex uncertainty set, while our model is valid for any type of polyhedral set.

2. In comparison to the approach in [7] and [14], our approach retains the convergence to the global optimum while allowing us to consider any type of polyhedral sets. The number of binary variables employed in the linearization of the complementarity conditions (6b) grows linearly with the number of inequality constraints used to define the uncertainty set. Hence, in the case where the uncertainty set is a multidimensional interval with a total budget for deviations, the number of binary variables is comparable to the one in the binary expansions in [7] and in [14]

\section{Results and Discussion}

In this section, we present results obtained by employing the proposed model on different application studies. Section 4.1 describes an example based on a simple two-node system, to illustrate the functioning of the model. A larger study based on the 24-node IEEE Reliability Test-System, the specifications of which can be found in [6], is considered in Section 4.2 .

\subsection{Illustrative Example}

Let us consider the two-node system depicted in Figure 1. The line between the two nodes of the system has a capacity of $60 \mathrm{MW}$ and a reactance of $0.13 \mathrm{pu}$. The load at each node is known with certainty and equal to $110 \mathrm{MWh}$ and $30 \mathrm{MWh}$ at node 1 and 2 , respectively, during the market period considered. Furthermore, two wind farms are located at each node. The day-ahead production forecast is equal to $20 \mathrm{MWh}$ and $25 \mathrm{MWh}$ for wind farms 1 and 2, respectively. Notice that we make the assumption that the length of a market period is one hour. Hence, a forecast production equal to $20 \mathrm{MWh}$ corresponds to an average output of $20 \mathrm{MW}$ during the considered period. However, for the sake of clarity we will hereinafter employ the unit MWh for energy production and dispatch, and MW for reserve and capacity.

The characteristics of the units in the system are listed in Table 1 . The capacity of each unit is indicated with $P^{\max }$, the per unit cost of production with $C$, while $C^{+}$and $C^{-}$represent the costs for each MW of available upward and downward reserve, respectively. Production from unit 1 is costly, but the unit is highly flexible, which is reflected in its low costs for reserve. On the contrary, unit 3 has a low marginal cost of production but is inflexible. Unit 2 has balanced characteristics. 


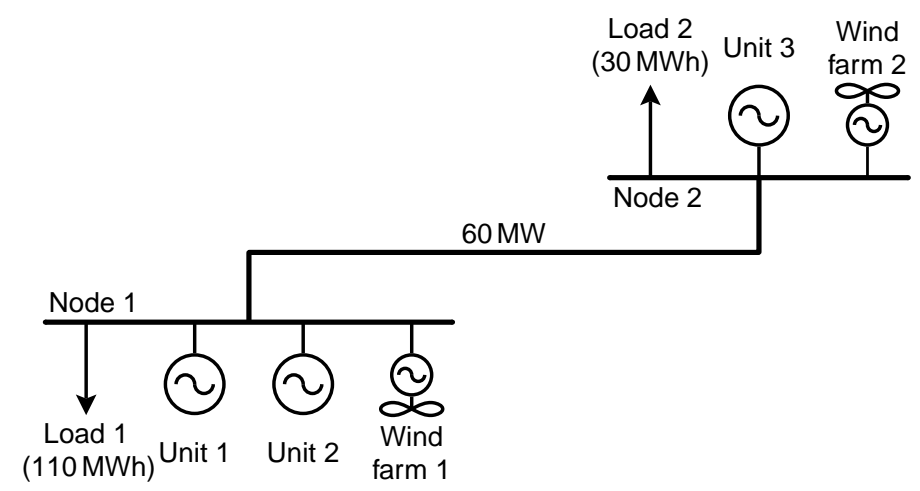

Figure 1: Two-node system

Table 1: Characteristics of the units in the two-node system

\begin{tabular}{lrrr}
\hline & Unit 1 & Unit 2 & Unit 3 \\
\hline$P^{\max }(\mathrm{MW})$ & 120 & 80 & 70 \\
$C(\$ / \mathrm{MWh})$ & 32 & 20 & 12 \\
$C^{+}(\$ / \mathrm{MW})$ & 7 & 11 & 15 \\
$C^{-}(\$ / \mathrm{MW})$ & 5 & 6 & 14 \\
\hline
\end{tabular}

Model (1) for this example writes as follows:

$$
\begin{aligned}
& \underset{\mathbf{p}, \mathbf{r}^{+}, \mathbf{r}^{-}, \boldsymbol{\delta}^{\mathbf{0}}}{\min } 32 p_{1}+20 p_{2}+12 p_{3}+7 r_{1}^{+}+11 r_{2}^{+}+15 r_{3}^{+}+5 r_{1}^{-}+6 r_{2}^{-}+14 r_{3}^{-}+\mathcal{Q}_{\mathcal{W}}\left(\mathbf{p}, \mathbf{r}^{+}, \mathbf{r}^{-}, \boldsymbol{\delta}^{\mathbf{0}}\right) \\
& \text { s.t. } p_{1}+p_{2}+20=110-\frac{\delta_{2}^{0}}{0.13} \\
& p_{3}+25=30+\frac{\delta_{2}^{0}}{0.13}, \\
& p_{1}+r_{1}^{+} \leq 120, \quad p_{2}+r_{2}^{+} \leq 80, \quad p_{3}+r_{3}^{+} \leq 70, \\
& p_{1}-r_{1}^{-} \geq 0 \quad, \quad p_{2}-r_{2}^{-} \geq 0 \quad, \quad p_{3}-r_{3}^{-} \geq 0 \text {, } \\
& -60 \leq \frac{\delta_{2}^{0}}{0.13} \leq 60 \\
& p_{1}, p_{2}, p_{3}, r_{1}^{+}, r_{2}^{+}, r_{3}^{+}, r_{1}^{-}, r_{2}^{-}, r_{3}^{-} \geq 0 \text {. }
\end{aligned}
$$

Constraints (11b) and (11c) enforce power balance at node 1 and 2, respectively. We choose node 1 as the reference node, i.e., we set $\delta_{1}^{0}=0$. Hence $-\delta_{2}^{0} / 0.13$ represents the energy flow from node 1 to node 2. Constraints (11d) ensure that the sum of the day-ahead dispatch $p$ and the upward reserve $r^{+}$is not greater than the maximum power output. Similarly, (11e) enforces that the scheduled downward reserve $r^{-}$is not greater than the dispatch $p$. As a result of (11f), the scheduled flow between the two nodes is within the transmission capacity. Finally, non-negativity of the variables is enforced by $(11 \mathrm{~g})$.

At the balancing stage, the actual wind power production can deviate from the day-ahead forecast. Table 2 reports forecast, maximum deviation (in absolute value) and the resulting minimum and maximum values of production for the two wind farms considered. We make the further assumption that production from the two wind farms cannot deviate by $\Delta w^{\max }$ at the same time. On the contrary, the sum between the ratios of their deviation divided by the relative $\Delta w^{\max }$ cannot be greater than $\Gamma=1.4$. Constant $\Gamma$ is referred to in the literature as the budget of uncertainty. Imposing this constraint implies that, if the deviation of one wind farm's production is equal to $\Delta w^{\max }$, then the other wind farm can deviate at most by $0.4 \times \Delta w^{\max }$.

Under the non restrictive assumption that the marginal cost for redispatch is equal to the day-ahead dispatch cost, and assuming a load-shedding cost equal to $\$ 200 / \mathrm{MWh}$, the worst-case recourse cost 
Table 2: Day-ahead forecast, maximum deviation at the balancing stage and resulting lower and upper bounds for production from each wind farm. Values in $M W h$

\begin{tabular}{ccccc}
\hline & $\widehat{w}$ & $\Delta w^{\max }$ & $\underline{w}$ & $\bar{w}$ \\
\hline Wind farm 1 & 20 & 15 & 5 & 35 \\
Wind farm 2 & 25 & 20 & 5 & 45 \\
\hline
\end{tabular}

$\mathcal{Q}_{\mathcal{W}}\left(\mathbf{p}, \mathbf{r}^{+}, \mathbf{r}^{-}, \boldsymbol{\delta}^{\mathbf{0}}\right)$ is equal to the objective function value of the following max-min problem, which corresponds to model (2):

$$
\begin{gathered}
\max _{\Delta \mathbf{w}} \underset{\substack{\mathbf{p}^{+}, \mathbf{p}^{-}, \mathbf{1}^{\mathrm{sh}}, \mathbf{w}^{\mathrm{sp}}, \boldsymbol{\delta}}}{\sin } 32\left(p_{1}^{+}-p_{1}^{-}\right)+20\left(p_{2}^{+}-p_{2}^{-}\right)+12\left(p_{3}^{+}-p_{3}^{-}\right)+200\left(l_{1}^{\mathrm{sh}}+l_{2}^{\mathrm{sh}}\right) \\
\text { s.t. } p_{1}^{+}-p_{1}^{-}+p_{2}^{+}-p_{2}^{-}+\Delta w_{1}-w_{1}^{\mathrm{sp}}+l_{1}^{\mathrm{sh}}=-\frac{\delta_{2}-\delta_{2}^{0}}{0.13} \\
p_{3}^{+}-p_{3}^{-}+\Delta w_{2}-w_{2}^{\mathrm{sp}}+l_{2}^{\mathrm{sh}}=\frac{\delta_{2}-\delta_{2}^{0}}{0.13} \\
p_{1}^{+} \leq r_{1}^{+}, \quad p_{2} \leq r_{2}^{+}, \quad p_{3} \leq r_{3}^{+}, \\
p_{1}^{-} \leq r_{1}^{-}, \quad p_{2}^{-} \leq r_{2}^{-}, \quad p_{3}^{-} \leq r_{3}^{-} \\
l_{1}^{\mathrm{sh}} \leq 110, \quad \quad_{2}^{\mathrm{sh}} \leq 30, \\
w_{1}^{\mathrm{sp}} \leq 20+\Delta w_{1}, \quad w_{2}^{\mathrm{sp}} \leq 25+\Delta w_{2} \\
-60 \leq \frac{\delta_{2}}{0.13} \leq 60, \\
p_{1}^{+}, p_{2}^{+}, p_{3}^{+}, p_{1}^{-}, p_{2}^{-}, p_{3}^{-}, l_{1}^{\mathrm{sh}}, l_{2}^{\mathrm{sh}}, w_{1}^{\mathrm{sp}}, w_{2}^{\mathrm{sp}} \geq 0 \\
\text { s.t. }-15 \leq \Delta w_{1} \leq 15, \quad-20 \leq \Delta w_{2} \leq 20 \\
\Delta w_{1}=\Delta w_{1}^{+}-\Delta w_{1}^{-}, \quad \Delta w_{2}=\Delta w_{2}^{+}-\Delta w_{2}^{-}, \\
\frac{\Delta w_{1}^{+}+\Delta w_{1}^{-}}{15}+\frac{\Delta w_{2}^{+}+\Delta w_{2}^{-}}{20} \leq 1.4
\end{gathered}
$$

The minimization problem enforces a minimum-cost redispatch in the electric energy system. Equations $(12 \mathrm{~b})$ and $(12 \mathrm{c})$ guarantee the nodal power balance at the balancing stage. Symbols $p^{+}$and $p^{-}$represent upward and downward redispatch, $\Delta w$ the deviation of wind power production from the day-ahead forecast, $w^{\mathrm{sp}}$ and $l^{\text {sh }}$ represent wind power spillage and load shedding, respectively, while $\delta$ represent the actual nodal network state. Constraints (12d) and (12e) enforce that the redispatch is not greater than the amount of upward and downward reserve established at the day-ahead stage, respectively. Constraints (12f) and (12g) guarantee that load shedding and wind power spillage are not greater than the actual consumption and wind power production. The actual transmission constraints are enforced by $(12 \mathrm{~h})$. Finally, non-negative variables are defined in (12i).

The maximization problem in model (12) picks the worst case realization of deviation $\Delta \mathbf{w}$ of the wind power production from the day-ahead forecast $\widehat{\mathbf{w}}$. The feasible space is polyhedral. Inequalities $(12 \mathrm{j})$ define the intervals over which the deviation of wind power production can occur. Equations (12k) split the deviation into its positive and negative parts $\Delta w^{+}$and $\Delta w^{-}$. Finally, constraint (12l) enforces the uncertainty budget.

Table 3(a) displays the results of model (11)-(12) in terms of day-ahead energy and reserve dispatch. These results can be compared to the ones obtained using a similar model based on stochastic programming and reported in Table 3(b). The latter model is obtained by replacing $\mathcal{Q}_{\mathcal{W}}(\cdot)$ in (11) with the expected value of the recourse cost over a discrete set of scenarios, rather than with its worst-case value determined by model (12). The scenarios used in the stochastic programming model are 100 random samples drawn from a uniform distribution defined over the uncertainty set (12j)-(121). While the day-ahead energy dispatch coincides, the following comments on reserve are in order:

1. As far as upward reserve is concerned, the robust optimization model yields more conservative results than the stochastic programming model. Indeed, no realization of the wind power deviation within 
the uncertainty set results in load-shedding events with the former approach. On the contrary, with the latter approach load shedding can take place if its cost is offset in expectation by the savings in terms of reserve.

2. No downward reserve is scheduled with the robust optimization approach. This is explained by the fact that there is no cost associated to wind power spillage. Hence, the worst-case realization of the wind power deviation is always negative, i.e., an underproduction, which requires no downward redispatch at the balancing stage.

3. With the robust optimization approach, units with the lowest aggregate cost of reserve and production, e.g., unit 3, are preferred to units with lower reserve cost but higher marginal costs, e.g., unit 1. This is a straightforward result of the focus on the worst-case realization of the uncertainty in (12). On the contrary, the stochastic programming model schedules reserve from units with lower capacity cost and higher operating costs, since the latter are weighted by the probability of actual deployment of the reserves.

Table 3: Results for day-ahead dispatch and reserve using the robust optimization and the stochastic programming approach

(a) Robust Optimization

\begin{tabular}{lrrr}
\hline & Unit 1 & Unit 2 & Unit 3 \\
\hline$p(\mathrm{MWh})$ & 0 & 30 & 65 \\
$r^{+}(\mathrm{MW})$ & 0 & 21 & 5 \\
$r^{-}(\mathrm{MW})$ & 0 & 0 & 0 \\
\hline
\end{tabular}

(b) Stochastic Programming

\begin{tabular}{lcrr}
\hline & Unit 1 & Unit 2 & Unit 3 \\
\hline$p(\mathrm{MWh})$ & 0 & 30 & 65 \\
$r^{+}(\mathrm{MW})$ & 9.60 & 8.47 & 0 \\
$r^{-}(\mathrm{MW})$ & 0 & 1.26 & 0 \\
\hline
\end{tabular}

In the following section, we assess the impact of the increased robustness of the proposed approach on the costs of dispatch, reserve and redispatch in a more realistic setup.

\subsection{Simulation Study}

The results presented in this section are obtained from a modified version of the 24-node IEEE Reliability Test-System in [6].

We include six wind farms at different locations throughout the grid. Table 4 reports the nodal location, as well as day-ahead forecast, maximum deviation at the balancing stage and the resulting minimum and maximum power production. The total forecast wind power production is $554 \mathrm{MWh}$. We consider the peak hour, where consumption totals $2850 \mathrm{MWh}$, thus wind power is expected to cover slightly less than $20 \%$ of the load.

Table 4: Nodal location, day-ahead forecast, maximum deviation at the balancing stage and resulting lower and upper bounds for production from each wind farm. Values in MWh

\begin{tabular}{ccrlll}
\hline Wind farm & Node & \multicolumn{1}{c}{$\widehat{w}$} & $\Delta w^{\max }$ & $\underline{w}$ & $\bar{w}$ \\
\hline 1 & 3 & 120 & 55.20 & 64.80 & 175.20 \\
2 & 5 & 96 & 40 & 56 & 136 \\
3 & 7 & 140 & 60 & 80 & 200 \\
4 & 16 & 52 & 52 & 0 & 104 \\
5 & 21 & 36 & 33.60 & 2.40 & 69.60 \\
6 & 23 & 110 & 66 & 44 & 176 \\
\hline
\end{tabular}

Besides the intervals reported in Table 4 and the budget constraint of the type of (12l), we introduce another type of linear constraints for the deviation of wind power production. The objective of these 
constraints is to limit the difference between deviations for adjacent wind farms, e.g., for units $q_{1}$ and $q_{2}$ :

$$
-\rho_{q_{1} q_{2}} \leq \frac{\Delta w_{q_{1}}}{\Delta w_{q_{1}}^{\max }}-\frac{\Delta w_{q_{2}}}{\Delta w_{q_{2}}^{\max }} \leq \rho_{q_{1} q_{2}} .
$$

We remark that solving the bilinear model (3) including constraints of this type is not straightforward, while in the proposed model (6) they can be included with little effort. Table 5 reports the values of $\rho$ employed in this study. Note that $\rho_{q_{1} q_{2}}$ is a measure of the spatial correlation between wind farms $q_{1}$ and $q_{2}$.

Table 5: Values of $\rho$ employed for constraints (13) in the 24-node system

\begin{tabular}{lcc}
\hline Wind farms & $\rho$ \\
\hline 1 & 2 & 0.4 \\
1 & 4 & 0.3 \\
2 & 3 & 0.5 \\
4 & 5 & 0.4 \\
5 & 6 & 0.5 \\
\hline
\end{tabular}

Capacity on the transmission lines connecting the node pairs $(15,21),(14,16)$ and $(13,23)$ is reduced to $400 \mathrm{MW}, 250 \mathrm{MW}$ and $250 \mathrm{MW}$, respectively. This is done in order to introduce bottlenecks in the transmission system. The production cost of the units is linearized by making use of a piecewise-linear approximation. Four blocks with constant marginal cost are employed for each power plant. As far as reserve provision is concerned, we assume that nuclear plants are totally inflexible and thus unable to provide reserve. For coal units, the reserve cost per MW is equal to one fourth of the marginal cost of its most expensive block. Oil units are assumed to be more flexible and their reserve cost is one tenth of the marginal cost of the most expensive block. Notice, however, that these units have the highest production cost among the plants considered. Finally, the load-shedding cost is set to $\$ 1000 / \mathrm{MWh}$.

\subsubsection{Comparison with the Stochastic Programming Approach.}

In this section we discuss the results in terms of system cost for the day-ahead dispatch and reserve schedules for the 24-node system, determined with the proposed robust optimization model and with a stochastic programming approach.

The uncertainty set in the robust optimization model is defined by the intervals in Table 4, constraints of the type (13) with the parameters defined according to Table 5 and a budget of uncertainty $\Gamma=3.5$.

For the model based on stochastic programming, 500 scenarios drawn from independent truncated Gaussian distributions model the wind power uncertainty at each site. The Gaussian distributions are scaled so that the upper and lower bounds in Table 4 represent the $95 \%$ confidence interval. Scenarios falling out of this interval, as well as the ones violating constraints (13) or exceeding the uncertainty budget are discarded.

Table 6 breaks down the system cost for the two approaches into day-ahead costs (for dispatch and reserve) and balancing costs (for redispatch and load-shedding). The latter are reported both in expectation, calculated over a validation set of 1000 scenarios drawn from the same distribution as the one used in the optimization of the stochastic programming model, and in the worst-case realization of the uncertainty within the set described above.

As one can notice, the reserve costs are comparable for the two approaches. Indeed, the higher level of conservatism of robust optimization is almost completely offset by the cost for downward reserve in the stochastic programming approach. However, the dispatch cost and, notably, the expected costs in the balancing stage are lower for the stochastic programming approach, which benefits from the possibility of redispatching downward rather than spilling wind power. In total, the stochastic programming approach outperforms the robust optimization one in terms of expected cost by about $2.8 \%$. However, the stochastic 
programming approach incurs a worst-case cost as high as three times the worst-case cost with the robust optimization model.

Table 6: Comparison of system cost with the robust optimization and the stochastic programming approaches. Values in \$

\begin{tabular}{|c|c|c|c|c|}
\hline Cost & \multicolumn{2}{|c|}{ Robust Optimization } & \multicolumn{2}{|c|}{ Stochastic Programming } \\
\hline Dispatch & \multicolumn{2}{|c|}{17897.52} & \multicolumn{2}{|c|}{17512.07} \\
\hline Upward reserve & \multicolumn{2}{|c|}{489.72} & \multicolumn{2}{|c|}{355.59} \\
\hline Downward reserve & & 0 & & 30.28 \\
\hline \multirow[t]{2}{*}{ Total day-ahead } & \multicolumn{2}{|c|}{18387.25} & \multicolumn{2}{|c|}{17997.95} \\
\hline & Expected & Worst-case & Expected & Worst-case \\
\hline Redispatch & 339.32 & 2989.46 & 147.82 & 2634.55 \\
\hline Load-shedding & 0 & 0 & 72.83 & 43586.85 \\
\hline Total balancing & 339.32 & 2989.46 & 220.64 & 46221.40 \\
\hline Total aggregate & 18726.56 & 21376.72 & 18218.59 & 64219.34 \\
\hline
\end{tabular}

\subsubsection{Robustness of Decision to Varying Distribution Type.}

The degree of suboptimality of the robust decision highlighted in the previous section might be an overestimation. In practice, the actual distribution of stochastic parameters can only be estimated by the decision-maker. Thus, the scenarios employed as input to stochastic programming models represent the uncertainty with a limited accuracy.

Table 7 illustrates the expectation of the system cost incurred by the robust optimization and by the stochastic programming models if the uncertainty has a different distribution than the one considered in Section 4.2.1. We now consider that deviations of wind power production at different locations follow independent uniform distributions defined on the same support as the Gaussian distribution employed in Section 4.2.1 to determine the stochastic programming solution. As one can notice, the expected value of system cost is rather stable if the solution obtained with the robust optimization model is employed. On the contrary, the cost incurred by the stochastic programming solution increases by roughly $\$ 1000$ compared to the one in Table 6, mostly owing to increasing load-shedding cost.

Table 7: Comparison of system cost with the robust optimization and the stochastic programming approaches under a different distribution. Values in $\$$

\begin{tabular}{lcc}
\hline Cost & Robust Optimization & Stochastic Programming \\
\hline Dispatch & 17897.52 & 17512.07 \\
Upward reserve & 489.72 & 355.59 \\
Downward reserve & 0 & 130.28 \\
Total day-ahead & 18387.25 & 17997.95 \\
Redispatch (exp.) & 576.75 & 335.06 \\
Load-shedding (exp.) & 0 & 923.26 \\
Total balancing (exp.) & 576.75 & 1258.32 \\
Total aggregate (exp.) & 18964.00 & 19256.27 \\
\hline
\end{tabular}

This comparison confirms the results in [2], which shows the stability of results of the robust approach to changes in the shape of the distribution. In practice, if the actual distribution of the uncertainty is more fat-tailed than the model from which scenarios are drawn, as in the case above, the performance of the stochastic programming solution degrades faster than the one of the robust optimization solution. 
For completeness of the analysis, we consider the inverse case, where scenarios drawn from a uniform distribution are used as input to the stochastic programming model. Then, the expected system cost is determined over a set of scenarios drawn from a normal distribution and truncated consistently with the uncertainty set. The expected system cost for the two approaches is reported in Table 8. Notably, the amount of reserve in the stochastic programming solution is significantly higher in this case than in the case in Table 6 where uncertainty is normally distributed. As a result, this solution is rather robust when the actual distribution of the uncertainty has lower weight on the tails, such as in this case. Indeed, no load-shedding events are observed, and the total expected system cost is lower than that for the robust optimization solution.

Table 8: Comparison of system cost with the robust optimization and the stochastic programming approaches under a different distribution. Values in $\$$

\begin{tabular}{lcc}
\hline Cost & Robust Optimization & Stochastic Programming \\
\hline Dispatch & 17897.52 & 17512.87 \\
Upward reserve & 489.72 & 433.42 \\
Downward reserve & 0 & 209.45 \\
Total day-ahead & 18387.25 & 18155.74 \\
Redispatch (exp.) & 361.10 & 139.36 \\
Load-shedding (exp.) & 0 & 0 \\
Total balancing (exp.) & 361.10 & 139.36 \\
Total aggregate (exp.) & 18748.34 & 18295.10 \\
\hline
\end{tabular}

\subsubsection{Computational Results.}

In this section, we briefly discuss the computational properties of the robust optimization and of the stochastic programming approaches. The models have been implemented in GAMS and solved using CPLEX 12, running on a laptop equipped with a 4-core processor clocking at $2.66 \mathrm{GHz}$.

Solution times for the 24-node system are illustrated in Figure 2. The robust optimization model converged in 29.11 s after four iterations of the cutting-plane algorithm. The solution time in this case is plotted as a horizontal dashed line in the figure since robust optimization is scenario-free. The solid line in the figure represents the time for solving the stochastic programming approach as a function of the number of scenarios. As one can see, the computation time for the stochastic programming model increases with the number of scenarios. The robust optimization model solves more quickly than the stochastic optimization one as soon as the number of scenarios is higher than 300 .

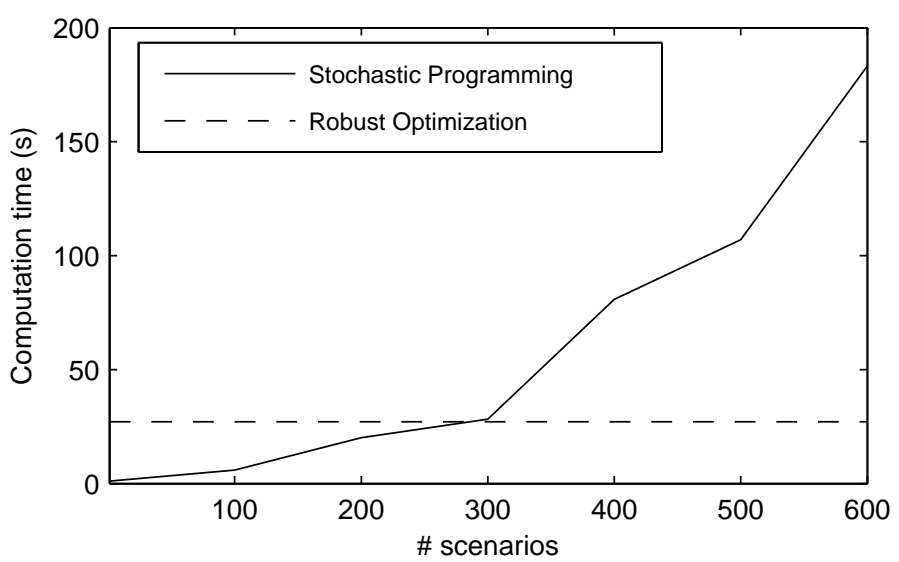

Figure 2: Computation time for the robust optimization approach, and for the stochastic programming approach as a function of the number of scenarios 


\section{Conclusions}

In this work, we consider the problem of jointly determining day-ahead energy dispatch and reserve capacity in an electricity market with large penetration of stochastic generation sources. The problem is cast as an adaptive robust optimization model, the solution of which minimizes the system cost in terms of dispatch, reserve and redispatch in the worst-case realization of the uncertain production.

We propose a novel reformulation of the problem that allows us to consider polyhedral sets of any type to model the support of the uncertainty distribution. Such a reformulation is employed as the subproblem within a well-known cutting-plane algorithm for adaptive robust optimization problems with right-hand side uncertainty. The proposed scheme, besides providing more flexibility in modeling uncertainty with general polyhedral sets, retains properties of convergence to the global optimum as well as of tractability of the methods proposed in the literature on the subject.

Through a case-study based on the 24-node IEEE Reliability Test-System, we assess the degree of suboptimality of the robust solution with respect to the stochastic programming approach. System cost increase in expectation by about $2.8 \%$. However, the robust solution cuts worst-case cost by over two thirds. Furthermore, we show that the proposed model is rather robust to changes in the probability distribution. Indeed, the robust solution can actually outperform the stochastic programming one if the model for the distribution of the uncertainty used in the latter one underestimates the probability density on the tails. Finally, we show that for the proposed model, the robust optimization approach has better computational properties than a reasonably sized equivalent stochastic programming model.

Future research on the topic should be carried out in different directions. Firstly, the complementary problem of modeling uncertainty sets for spatially distributed stochastic production, which is not considered here, should be studied. In parallel, dynamic properties could also be considered both in terms of modeling uncertainty sets, and by extending the model to a multi-period setting. Furthermore, reformulations of the robust optimization problem should be thought of in order to account for uncertainty sets with different structure, e.g., ellipsoidal sets. Finally, the combination of the proposed model with methods for determining ex-post the amount of downward reserve should be investigated as well, in order to reduce the gap of the robust approach with the stochastic programming one in terms of expected system cost.

\section{A Complete Min-Max-Min Optimization Model}

A description of the symbols employed in these appendices is included in Table 9.

The complete min-max-min model used in this work to determine the robust energy and reserve dispatch is the following optimization problem:

$$
\begin{array}{rlr}
\min _{\mathbf{p}, \mathbf{r}^{+}, \mathbf{r}^{-}, \delta^{\mathbf{0}}} & \sum_{k} C_{k} p_{k}+C_{k}^{+} r_{k}^{+}+C_{k}^{-} r_{k}^{-}+\mathcal{Q}_{\mathcal{W}}\left(\mathbf{p}, \mathbf{r}^{+}, \mathbf{r}^{-}, \boldsymbol{\delta}^{\mathbf{0}}\right) & \\
\text { s.t. } & \sum_{k \in G_{i}} p_{k}=\sum_{j \in N_{i}} B_{i j}\left(\delta_{i}^{0}-\delta_{j}^{0}\right)+d_{s(i)}-\widehat{w}_{q(i)}, & \\
& \delta_{1}^{0}=0, & \\
& p_{k}+r_{k}^{+} \leq P_{k}^{\max }, & \\
& p_{k}-r_{k}^{-} \geq 0, & \forall k, \\
& B_{i j}\left(\delta_{i}^{0}-\delta_{j}^{0}\right) \leq T_{i j}^{\max }, & \forall k, \\
& p_{k}, r_{k}^{+}, r_{k}^{-} \geq 0, & \forall i, j \in N_{i}, \\
\end{array}
$$

where the worst-case recourse cost for energy redispatching at the balancing stage is yielded by the 
Table 9: List of symbols

\begin{tabular}{|c|c|c|}
\hline Symbol & Type & Description \\
\hline$k$ & index & index for production block \\
\hline$i, j$ & index & index for network node \\
\hline$q, u$ & index & index for wind farm \\
\hline$s$ & index & index for load \\
\hline$G_{i}$ & set & set of production blocks $k$ at node $i$ \\
\hline$N_{i}$ & set & set of nodes $j$ connected by a transmission line to node $i$ \\
\hline$W_{q}$ & set & set of wind farms $u$ neighboring wind farm $q$ \\
\hline$s(i)$ & set & load at node $i$ of the network (if any) \\
\hline$q(i)$ & set & wind farm at node $i$ of the network (if any) \\
\hline$i(k)$ & set & node where production block $k$ is located \\
\hline$i(s)$ & set & node where load $s$ is located \\
\hline$i(q)$ & set & node where wind farm $q$ is located \\
\hline$C_{k}$ & parameter & per unit cost of energy dispatch and redispatch for unit $k$ \\
\hline$C_{k}^{+}$ & parameter & per unit cost of upward reserve for unit $k$ \\
\hline$C_{k}^{-}$ & parameter & per unit cost of downward reserve for unit $k$ \\
\hline$d_{s}$ & parameter & load from consumer $s$ \\
\hline$\widehat{w}_{q}$ & parameter & forecast wind power production for wind farm $q$ \\
\hline$B_{i j}$ & parameter & susceptance of line connecting nodes $i$ and $j$ \\
\hline$T_{i j}^{\max }$ & parameter & capacity of line connecting nodes $i$ and $j$ \\
\hline$V^{\text {sh }}$ & parameter & per unit cost of load-shedding \\
\hline$\Delta w_{q}^{\max }$ & parameter & maximum forecast error for output of wind farm $q$ \\
\hline & parameter & budget of uncertainty for forecast error of wind power production \\
\hline$\rho_{q u}$ & parameter & maximum deviation of forecast error between wind farms $q$ and $u$ \\
\hline$p_{k}$ & variable & energy dispatch for production block $k$ \\
\hline$r_{k}^{+}$ & variable & upward reserve dispatch for production block $k$ \\
\hline$r_{k}^{-}$ & variable & downward reserve dispatch for production block $k$ \\
\hline$\delta_{i}^{0}$ & variable & network state variable at node $i$ at the day-ahead stage \\
\hline$\Delta w_{q}$ & variable & forecast error for production from wind farm $q$ \\
\hline$\Delta w_{q}^{+}$ & variable & positive part of forecast error for production from wind farm $q$ \\
\hline$\Delta w_{q}^{-}$ & variable & negative part of forecast error for production from wind farm $q$ \\
\hline$p_{k}^{+}$ & variable & upward energy redispatch for production block $k$ \\
\hline$p_{k}^{-}$ & variable & downward energy redispatch for production block $k$ \\
\hline$l_{s}^{\mathrm{sh}}$ & variable & load shedding for demand $s$ \\
\hline$w_{q}^{\mathrm{sp}}$ & variable & wind power spillage for wind farm $q$ \\
\hline$\delta_{i}$ & variable & network state variable (voltage angle) at node $i$ at the balancing stage \\
\hline
\end{tabular}


following max-min optimization model:

$$
\begin{aligned}
& \mathcal{Q}_{\mathcal{W}}\left(\mathbf{p}, \mathbf{r}^{+}, \mathbf{r}^{-}, \boldsymbol{\delta}^{\mathbf{0}}\right)= \\
& \max _{\boldsymbol{\Delta} \mathbf{w}, \boldsymbol{\Delta} \mathbf{w}^{+}, \Delta \mathbf{w}^{-}} \min _{\substack{\mathbf{p}^{+}, \mathbf{p}^{-} \\
\mathbf{l}^{\mathrm{sh}}, \mathbf{w}^{\mathbf{s p}}, \boldsymbol{\delta}}} \sum_{k} C_{k}\left(p_{k}^{+}-p_{k}^{-}\right)+\sum_{s} V^{\mathrm{sh}} l_{s}^{\mathrm{sh}} \\
& \text { s.t. } \sum_{k \in G_{i}}\left(p_{k}^{+}-p_{k}^{-}\right)+l_{s(i)}^{\mathrm{sh}}+\Delta w_{q(i)}-w_{q(i)}^{\mathrm{sp}}= \\
& \sum_{j \in N_{i}} B_{i j}\left(\delta_{i}-\delta_{i}^{0}-\delta_{j}+\delta_{j}^{0}\right), \\
& \delta_{1}=0, \quad: \nu, \\
& p_{k}^{+} \leq r_{k}^{+}, \quad: \mu_{k}^{+}, \quad \forall k, \\
& p_{k}^{-} \leq r_{k}^{-}, \quad: \mu_{k}^{-}, \quad \forall k, \\
& l_{s}^{\mathrm{sh}} \leq d_{s}, \quad: \epsilon_{s}^{\mathrm{sh}}, \quad \forall s, \\
& w_{q}^{\mathrm{sp}} \leq \widehat{w}_{q}+\Delta w_{q}, \quad: \epsilon_{q}^{\mathrm{sp}}, \quad \forall q, \\
& B_{i j}\left(\delta_{i}-\delta_{j}\right) \leq T_{i j}^{\max } \quad: \sigma_{i j}, \quad \forall i, j \in N_{i} \text {, } \\
& p_{k}^{+}, p_{k}^{-} \geq 0, \forall k, l_{s}^{\mathrm{sh}} \geq 0, \forall s, w_{q}^{\mathrm{sp}} \geq 0, \forall q, \\
& \text { s.t. } \Delta w_{q}=\Delta w_{q}^{+}-\Delta w_{q}^{-}, \quad \forall q \text {, } \\
& \Delta w_{q}^{+} \leq \Delta w_{q}^{\max }, \quad \forall q, \\
& \Delta w_{q}^{-} \leq \Delta w_{q}^{\max }, \quad \forall q, \\
& \sum_{q} \frac{\Delta w_{q}^{+}+\Delta w_{q}^{-}}{\Delta w_{q}^{\max }} \leq \Gamma, \\
& \frac{\Delta w_{q}}{\Delta w_{q}^{\max }}-\frac{\Delta w_{u}}{\Delta w_{u}^{\max }} \leq \rho_{q u}, \quad \forall q, u \in W_{q}, \\
& \Delta w_{q}^{+}, \Delta w_{q}^{-} \geq 0, \quad \forall q .
\end{aligned}
$$

Notice that (14) is the complete formulation of (1) for the problem at hand. Similarly, (15) corresponds to $(2)$.

\section{B Optimization Model Used Within the Cutting-Plane Algo- rithm}

In this section, we carry out in detail the mathematical development that leads to the single-level maximization problem (6).

Let us first replace the right-hand side minimization problem in (15) with its dual. Notice that the dual variables are indicated in (15) after a colon separating them from the corresponding constraints. We then swap the order of the resulting max-max problem, so that the right-hand-side maximization problem is the one involving variables $\Delta \mathrm{w}, \Delta \mathbf{w}^{+}, \Delta \mathbf{w}^{-}$. These operations render the following problem:

$$
\begin{aligned}
\max _{\substack{\boldsymbol{\lambda}, \nu, \boldsymbol{\mu}^{+}, \boldsymbol{\mu}^{-} \\
\boldsymbol{\epsilon}^{\mathrm{sh}}, \boldsymbol{\epsilon}^{\mathrm{sp}}, \boldsymbol{\sigma}}} \sum_{i}-\lambda_{i} \sum_{j \in N_{i}} B_{i j}\left(\delta_{i}^{0}-\delta_{j}^{0}\right)+\sum_{k}\left(\mu_{k}^{+} r_{k}^{+}+\mu_{k}^{-} r_{k}^{-}\right) \\
+\sum_{s} d_{s} \epsilon_{s}^{\mathrm{sh}}+\sum_{q} \widehat{w}_{q} \epsilon_{q}^{\mathrm{sp}}+\sum_{i, j \in N_{i}} T_{i j}^{\max } \sigma_{i j} \\
+\max _{\Delta \mathbf{w}, \Delta \mathbf{w}^{+}, \Delta \mathbf{w}^{-}}-\sum_{i} \lambda_{i} \Delta w_{q(i)}+\sum_{q} \epsilon_{q}^{\mathrm{sp}} \Delta w_{q} \\
\text { s.t. } \Delta w_{q}=\Delta w_{q}^{+}-\Delta w_{q}^{-} \quad: \gamma_{q}, \quad \forall q,
\end{aligned}
$$




$$
\begin{aligned}
& \Delta w_{q}^{+} \leq \Delta w_{q}^{\max } \quad: \xi_{q}^{+}, \quad \forall q \\
& \Delta w_{q}^{-} \leq \Delta w_{q}^{\max } \quad: \xi_{q}^{-}, \quad \forall q, \\
& \sum_{q} \frac{\Delta w_{q}^{+}+\Delta w_{q}^{-}}{\Delta w_{q}^{\max }} \leq \Gamma \quad: \eta, \\
& \frac{\Delta w_{q}}{\Delta w_{q}^{\max }}-\frac{\Delta w_{u}}{\Delta w_{u}^{\max }} \leq \rho_{q u} \quad: \omega_{q u}, \quad \forall q, u \in W_{q}, \\
& \Delta w_{q}^{+}, \Delta w_{q}^{-} \geq 0 \quad \forall q, \\
& \text { s.t. } \lambda_{i(k)}+\mu_{k}^{+} \leq C_{k} \\
& -\lambda_{i(k)}+\mu_{k}^{-} \leq-C_{k}, \\
& \forall k, \\
& \forall k, \\
& \lambda_{i(s)}+\epsilon_{s}^{\mathrm{sh}} \leq V^{\mathrm{sh}} \text {, } \\
& -\lambda_{i(q)}+\epsilon_{q}^{\mathrm{sp}} \leq 0 \\
& \forall s, \\
& \forall q, \\
& \nu-\left(\sum_{j \in N_{1}} B_{1 j}\right) \lambda_{1}+\left(\sum_{j \in N_{1}} B_{1 j} \lambda_{j}\right)+\sum_{j \in N_{1}} B_{1 j}\left(\sigma_{1 j}-\sigma_{j 1}\right)=0, \\
& -\left(\sum_{j \in N_{i}} B_{i j}\right) \lambda_{i}+\left(\sum_{j \in N_{i}} B_{i j} \lambda_{j}\right)+\sum_{j \in N_{i}} B_{i j}\left(\sigma_{i j}-\sigma_{j i}\right)=0, \forall i \neq 1, \\
& \mu_{k}^{+}, \mu_{k}^{-} \leq 0, \forall k, \epsilon_{s}^{\mathrm{sh}} \leq 0, \forall s, \epsilon_{q}^{\mathrm{sp}} \leq 0, \forall q, \sigma_{i j} \leq 0, \forall i, j \in N_{i},
\end{aligned}
$$

which is the complete formulation of the mid and right-hand-side maximization problems in (4).

Then, we replace the objective function of the right-hand-side maximization problem in (16) with the one of its dual problem:

$$
-\sum_{i} \lambda_{i} \Delta w_{q(i)}+\sum_{q} \epsilon_{q}^{\mathrm{sp}} \Delta w_{q}=\sum_{q} \Delta w_{q}^{\max }\left(\xi_{q}^{+}+\xi_{q}^{-}\right)+\Gamma \eta+\sum_{q, u \in W_{q}} \rho_{q u} \omega_{q u} .
$$

Finally, we include the Karush-Kuhn-Tucker conditions for the right-hand-side optimization problem, and cast (16) as the following single-level maximization problem:

$$
\begin{aligned}
& \max _{\substack{\boldsymbol{\lambda}, \nu, \boldsymbol{\mu}^{+}, \boldsymbol{\mu}^{-}, \epsilon^{\mathrm{sh}}, \boldsymbol{\epsilon}^{\mathrm{sp}}, \boldsymbol{\Delta} \mathbf{\Delta} \mathbf{w}^{+}, \boldsymbol{\Delta} \mathbf{w}^{-}, \boldsymbol{\gamma}, \boldsymbol{\xi}^{+}, \boldsymbol{\xi}^{-}, \eta, \boldsymbol{\omega}}} \sum_{i}-\lambda_{i} \sum_{j \in N_{i}} B_{i j}\left(\delta_{i}^{0}-\delta_{j}^{0}\right)+\sum_{k}\left(\mu_{k}^{+} r_{k}^{+}+\mu_{k}^{-} r_{k}^{-}\right) \\
& +\sum_{s} d_{s} \epsilon_{s}^{\mathrm{sh}}+\sum_{q} \widehat{w}_{q} \epsilon_{q}^{\mathrm{sp}}+\sum_{i, j \in N_{i}} T_{i j}^{\max } \sigma_{i j} \\
& +\sum_{q} \Delta w_{q}^{\max }\left(\xi_{q}^{+}+\xi_{q}^{-}\right)+\Gamma \eta+\sum_{q, u \in W_{q}} \rho_{q u} \omega_{q u} \\
& \text { s.t. } \Delta w_{q}=\Delta w_{q}^{+}-\Delta w_{q}^{-} \text {, } \\
& 0 \leq \xi_{q}^{+} \perp \Delta w_{q}^{+}-\Delta w_{q}^{\max } \leq 0, \\
& 0 \leq \xi_{q}^{-} \perp \Delta w_{q}^{-}-\Delta w_{q}^{\max } \leq 0, \\
& 0 \leq \eta \perp \sum_{q} \frac{\Delta w_{q}^{+}+\Delta w_{q}^{-}}{\Delta w_{q}^{\max }}-\Gamma \leq 0, \\
& 0 \leq \omega_{q u} \perp \frac{\Delta w_{q}}{\Delta w_{q}^{\max }}-\frac{\Delta w_{u}}{\Delta w_{u}^{\max }}-\rho_{q u} \leq 0, \\
& \gamma_{q}+\sum_{u \in W_{q}} \frac{\omega_{q u}-\omega_{u q}}{\Delta w_{q}^{\max }}+\lambda_{q(i)}-\epsilon_{q}^{\mathrm{sp}}=0 \\
& 0 \leq \Delta w_{q}^{+} \perp-\gamma_{q}+\xi_{q}^{+}+\frac{\eta}{\Delta w_{q}^{\max }} \geq 0,
\end{aligned}
$$




$$
\begin{array}{ll}
0 \leq \Delta w_{q}^{-} \perp \gamma_{q}+\xi_{q}^{-}+\frac{\eta}{\Delta w_{q}^{\max } \geq 0,} & \forall q, \quad(18 \mathrm{k}) \\
\lambda_{i(k)}+\mu_{k}^{+} \leq C_{k}, & \forall k, \quad(18 \mathrm{l}) \\
-\lambda_{i(k)}+\mu_{k}^{-} \leq-C_{k}, & \forall k, \quad(18 \mathrm{~m}) \\
\lambda_{i(s)}+\epsilon_{s}^{\mathrm{sh}} \leq V^{\mathrm{sh}}, & \forall s, \quad(18 \mathrm{n}) \\
-\lambda_{i(q)}+\epsilon_{q}^{\mathrm{sp}} \leq 0, & \forall q, \quad(18 \mathrm{o}) \\
\nu-\left(\sum_{j \in N_{1}} B_{1 j}\right) \lambda_{1}+\left(\sum_{j \in N_{1}} B_{1 j} \lambda_{j}\right)+\sum_{j \in N_{1}} B_{1 j}\left(\sigma_{1 j}-\sigma_{j 1}\right)=0, & \\
-\left(\sum_{j \in N_{i}} B_{i j}\right) \lambda_{i}+\left(\sum_{j \in N_{i}} B_{i j} \lambda_{j}\right)+\sum_{j \in N_{i}} B_{i j}\left(\sigma_{i j}-\sigma_{j i}\right)=0, & \forall i \neq 1 \\
\mu_{k}^{+}, \mu_{k}^{-} \leq 0, \forall k, \epsilon_{s}^{\mathrm{sh}} \leq 0, \forall s, \epsilon_{q}^{\mathrm{sp}} \leq 0, \forall q, \sigma_{i j} \leq 0, \forall i, j \in N_{i} &
\end{array}
$$

which is the complete formulation of the right-hand side maximization problem in (6).

\section{References}

[1] D. Bertsimas, D. G. Brown, and C. Caramanis. Theory and applications of robust optimization. SIAM Review, 53(3):464-501, 2011.

[2] D. Bertsimas, E. Litvinov, X. A. Sun, J. Zhao, and T. Zheng. Adaptive robust optimization for the security constrainted unit commitment problem. IEEE Transactions on Power Systems, 28(1):52-63, 2012.

[3] A. J. Conejo, E. Castillo, R. Mínguez, and R. García-Bertrand. Decomposition Techniques in Mathematical Programming: Engineering and Science Applications, chapter 5, pages 197-199. Springer, Berlin, 2006.

[4] J. Fortuny-Amat and B. McCarl. A representation and economic interpretation of a two-level programming problem. Journal of the Operational Research Society, pages 783-792, 1981.

[5] S. A. Gabriel, A. J. Conejo, J. D. Fuller, B. F. Hobbs, and C. Ruiz. Complementarity Modeling in Energy Markets, volume 180 of International Series in Operations Research $\&$ Management Science, chapter 6, pages 221-262. Springer, New York, 2012.

[6] C. Grigg, P. Wong, P. Albrecht, R. Allan, M. Bhavaraju, R. Billinton, Q. Chen, C. Fong, S. Haddad, S. Kuruganty, et al. The IEEE reliability test system-1996. A report prepared by the reliability test system task force of the application of probability methods subcommittee. IEEE Transactions on Power Systems, 14(3):1010-1020, 1999.

[7] R. Jiang, J. Wang, and Y. Guan. Robust unit commitment with wind power and pumped storage hydro. IEEE Transactions on Power Systems, 27(2):800-810, May 2012.

[8] J. E. Kelley. The cutting-plane method for solving convex programs. SIAM Journal of the Society for Industrial and Applied Mathematics, 8(4):703-712, 1960.

[9] J. M. Morales, A. J. Conejo, and J. Pérez-Ruiz. Economic valuation of reserves in power systems with high penetration of wind power. IEEE Transactions on Power Systems, 24(2):900-910, 2009.

[10] G. Pritchard, G. Zakeri, and A. Philpott. A single-settlement, energy-only electric power market for unpredictable and intermittent participants. Operations Research, 58(4(2)):1210-1219, 2010.

[11] S. Siddiqui and S. A. Gabriel. An SOS1-based approach for solving MPECs with a natural gas market application. Networks and Spatial Economics, pages 1-23, 2012.

[12] A. Thiele, T. Terry, and M. Epelman. Robust linear optimization with recourse. IOE Technical Report TR09-01, University of Michigan, mar 2010.

[13] B. Zeng and L. Zhao. Solving two-stage robust optimization problems by a column-and-constraint generation method. Technical report, Department of Industrial and Management Syst. Engineering, University of South Florida, 2011.

[14] L. Zhao and B. Zeng. Robust unit commitment problem with demand response and wind energy. In 2012 IEEE Power \& Energy Society General Meeting, pages 1-8, 2012. 